\title{
Costos Estimados en el 2010 para Establecer y Producir Pitaya (Fruta Dragón) en el Sur de Florida ${ }^{1}$
}

\author{
Edward A. Evans, Jordan Huntley, Jonathan Crane, and Allen F. Wysocki
}

\section{Introducción}

Como consecuencia de la creciente competencia extranjera y la disminución de rendimientos de los productos agrícolas tradicionales, muchos productores en el Sur de la Florida se han embarcado en una búsqueda agresiva de productos agrícolas que sean alternativas viables. Un producto que ha llamado la atención es la pitaya (género Hylocereus, también conocida como fruta de dragón [Figura 1]), una especie de cactus trepador autóctona de las regiones de bosques tropicales de México, Centroamérica y América del Sur (Mizrahi, Nerd y Nobel 1997). De menos de 50 hectáreas plantadas en la Florida en fecha tan reciente como 2006 (Steele y Crane 2006), la producción se ha multiplicado por seis y ahora se estima en alrededor de 320 acres (comunicación personal, Jonathan Crane).

Varios factores son responsables por la atención que se le da a este cultivo, tales como la promesa de altos retornos netos basados en el aumento de la población Asiática en los Estados Unidos, los beneficios para la salud asociados con los altos contenidos de antioxidantes de la fruta y la popularidad de la fruta en restaurantes de alta calidad, debido
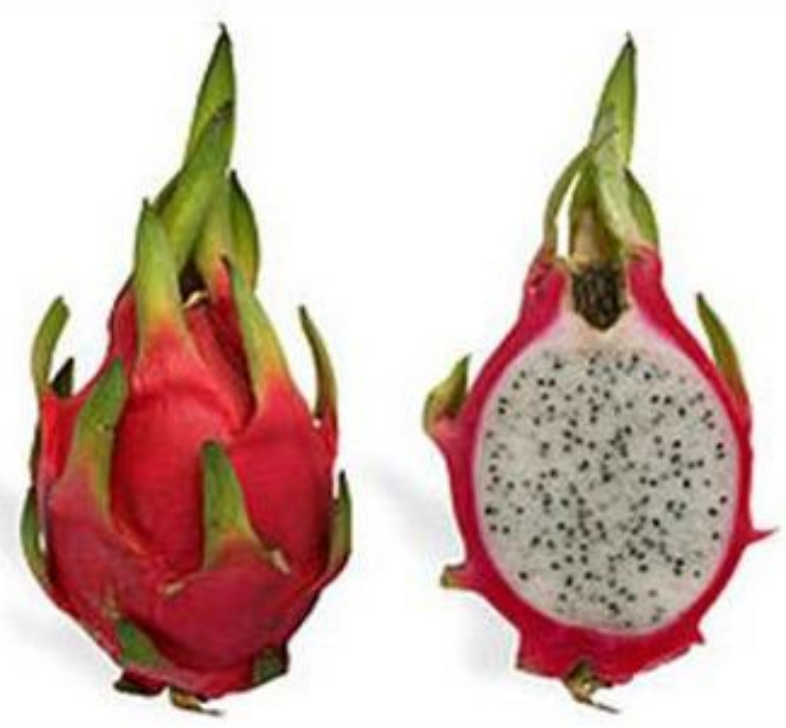

Figure 1. Pitaya (fruta dragón)

a su sabor único, su belleza y versatilidad (se puede comer fresco o procesado en el jugo, postres, mermeladas, helados, cócteles y vino). Como resultado de todo esto, la pitaya se ha popularizado con los productores y los consumidores (Lobo y Bender 2008).

1. Este documento EDIS, FE921 (traducción al Español de FE888), es una publicación del Departamento de Food and Resource Economics, Florida Cooperative Extension Service, Institute of Food and Agricultural Sciences, University of Florida, Gainesville, FL. Publicada en marzo del 2013. La traducción del inglés al español estuvo a cargo de Carlos Jáuregui. Por favor, visite el sitio de EDIS en la red en http://edis.ifas.ufl.edu.

2. Edward A. Evans, professor asociado, Food and Resource Economics Department, University of Florida, Tropical Research and Education Center, Homestead, FL; Jordan Huntley, graduado con maestría en Agribusiness, Food and Resource Economics Department, University of Florida, Tropical Research and Education Center, Homestead, FL; Jonathan Crane, profesor, Horticultural Sciences Department, University of Florida, Tropical Research and Education Center, Homestead, FL; and Allen Wysocki, decano asociado y professor asociado, Food and Resource Economics Department, University of Florida, Gainesville, FL; Florida Cooperative Extension Service, Institute of Food and Agricultural Sciences, University of Florida, Gainesville, FL.

El uso de marcas registradas en esta publicación es solo con el propósito de proveer información específica. UF/IFAS no garantiza los productos mencionados y referencias a ellos en esta publicación no significa respaldo de estos productos o la exclusion de otros productos similares. 
La pitaya también exhibe algunas características agronómicas distintas y deseables que aumentan sus perspectivas como cultivo comercial substituto adecuado y viable. Estas características incluyen la relativa facilidad con la que se pueden propagar (por estacas), reduciendo así el gasto que normalmente se asocia con la compra de materiales adicionales de plantación; el costo relativamente bajo de mantenimiento del cultivo, el tiempo corto entre la siembra y la cosecha (12-18 meses) en comparación a otros árboles frutales tradicionales, y su tasa de alto rendimiento, que va de 20 a 60 libras por planta (Gunasena, Pushpakumara y Kariyawasam 2006). Además, la pitaya es un cultivo perenne, con una vida útil de 20 a 30 años, asegurando que, con un cuidado adecuado, puede proporcionar un flujo constante de ingresos (Crane y Balerdi 2009; Gunasena, Pushpakumara y Kariyawasam 2006).

En el lado negativo, hay necesidad de más información sobre los costos de establecimiento y mantenimiento de huertos de pitaya así como de su rentabilidad (Crane and Balerdi 2009; Gunasena, Pushpakumara, and Kariyawasam 2006). Consecuentemente, el objetivo de este artículo es el de proveer información sobre los costos y retornos asociados con el establecimiento y mantenimiento de un huerto de cinco acres de pitaya en el sur de Florida, así como el de establecer los precios y rendimientos que se deben obtener para que el huerto de pitaya sea rentable. Por lo tanto, nuestro enfoque es más sobre el lado económico que agronómico. Las personas interesadas en más información sobre el aspecto agronómico de producción de la pitaya pueden leer el documento HS1068 de EDIS, Pitaya Growing in the Florida Home Landscape (Crane and Balerdi 2009).

\section{Enfoque y Asunciones Principales del Análisis}

Información usada en el análisis fue obtenida a través de entrevistas con productores, operadores de viveros, empacadores, proveedores de productos químicos y proveedores de otros insumos agrícolas en el sur de Florida. Los investigadores prepararon un cuestionario que incluye preguntas pertinentes al establecimiento y operación de un huerto de pitaya. La información fue recolectada en el verano del 2010 a través de entrevistas personales y por teléfono. Además de las entrevistas, información fue obtenida a través de los agentes de extensión de la Universidad de Florida así come de la literatura existente. Las asunciones usadas en el modelo son las siguientes:

- Terreno-Debido a que la mayoría de productores en el sur de Florida son dueños del terreno, se usó un costo de arrendamiento de $\$ 500$ por acre para tomar en cuenta el costo de oportunidad del terreno. El presupuesto y costos de producción están basados en un huerto de cinco acres (tamaño mínimo permitido).

- Diseño del Huerto-Se pusieron enrejados a una distancia de 5 pies por 15 pies ( 5 pies entre enrejados de la misma la fila y 15 pies entre filas), por un total de 581 enrejados por acre. Se pusieron tres plantas por cada enrejado por un total de 1,743 plantas por acre.

- Sistema de Enrejado-Para este análisis, los enrejados se hicieron usando postes de 8 pies de largo por 5 pulgadas cuadradas de grueso que se plantaron a una profundidad de 2 pies y que pasaron a través del centro de un contenedor de 65 galones, llenado con 6.7 pies de tierra para macetas. Para cada enrejado, se taladraron dos orificios en la parte superior del poste. Dos barras de refuerzo \# 3 de 2 pies se insertaron en los orificios y un alambre de cercado de 4 pies de largo se aseguró a cada barra de refuerzo. El costo estimado por enrejado fue de \$32.

- Variedades-Se escogió la especie Vietnamés de pulpa blanca (Hylocereus undatus) porque produce un gran volumen de fruta.

- Fertilización-La fertilización consiste de 1,188 libras del fertilizante 8-3-9 cuatro veces al año y de un tratamiento al año de 1.5 galones por acre con el fertilizante foliar (16-16-8).

- Manejo de Hierbas-El control de hierbas requiere varias fumigaciones al año. Para el control de hierbas dentro de las filas se aplicaron, cuatro veces al año, 0.5 galones por acre de un herbicida a base de glifosatos. Dentro de los contenedores, se hicieron cuatro aplicaciones al año con el herbicida pre-emergente Ronstar ${ }^{\bullet}$, a razón de 45 libras por acre, por aplicación, por año. Las hierbas en las filas también fueron cortadas cuatro a seis veces al año por un costo total de $\$ 120$ por acre.

- Manejo de Pestes-Los tratamientos con pesticidas incluyen cuatro aplicaciones de 50 libras por acre de "slug and snail bait" (cebo para babosas y caracoles) y una aplicación de una libra por acre de Malathion ${ }^{\circledR}$. Muchos de los productores también usan fungicidas.

- Irrigación-Muchos de los productores instalan sistemas de aspersión debido a los altos requerimientos de agua, a pesar de que las plantas pertenecen a la familia cactácea. Los costos de irrigación, incluyendo los costos de 
bombeo de agua y labor de mantenimiento se estimaron en $\$ 50$ por acre, por año.

- Salarios-Se asumió un salario de $\$ 15.00$ por hora para los trabajadores calificados y de $\$ 11.00$ para los trabajadores de campo. Estos pagos incluyen las contribuciones del empleador a beneficios como compensación del trabajador, seguro social, impuestos a medicare y seguro de salud. En la cosecha, el pago fue de acuerdo a la cantidad cosechada.

- Cosecha y Costos de Mercadeo-Se asumió que el costo de cosecha y transporte de la fruta de pitaya fue de $\$ 0.05$ por libra.

- Rendimientos-Se pueden obtener cantidades significantes de pitaya a partir del segundo año de producción. En base a la información proveída por los productores, los rendimientos promedios que se pueden vender se calcularon de la siguiente manera: primer año cero libras por acre; segundo año, 10,000 libras por acre; tercer año, 15,000 libras por acre; $y$ años de producción plena (del cuarto año en adelante), 19,000 libras por acre.

- Precios de la Pitaya-Se estimó que el precio promedio de la pitaya fue de $\$ 1.35$ por libra. Este estimado se hizo usando el precio que las empacadoras del sur de Florida pagaron durante los meses de Julio a Septiembre del 2009. Los precios oscilaron entre $\$ 0.85$ y $\$ 2.50$ por libra.

- Intereses sobre los Costos de Pre-Cosecha-Esto refleja el costo de los préstamos de dinero o el costo de oportunidad de la propiedad. En los cálculos se usó una tasa nominal del cinco por ciento.

- Cargos por Maquinaria y Equipo-Los cargos por maquinaria y equipos (recobro de costos de capital) se calcularon en base a la vida esperada del equipo o maquinaria, valor de rescate, precio de compra y una tasa de interés del cinco por ciento. Estos cargos representan la cantidad de dinero que la empresa paga por el uso de los bienes de tal manera que el valor del bien será recobrado dentro de un tiempo específico a una designada tasa de interés.

- Amortización de los Costos de Establecimiento-Debido a que los primeros tres años son considerados como la fase de establecimiento, se asumió que el huerto es considerado maduro después del tercer año. Los costos acumulados en los tres años se amortizan sobre la vida del huerto (que se asumen son de 20 años) y se le carga a la empresa como parte del costo fijo.
- Interés Acumulado Sobre los Costos de EstablecimientoEsto representa los cargos acumulados sobre los préstamos o sobre los retornos no recibidos durante la fase de establecimiento. Se aplicó una tasa de interés nominal del cinco por ciento.

\section{Resumen de los Resultados}

Basados en las asunciones proveídas en este estudio, los costos estimados para establecer un huerto de pitaya (Cuadro 1) es de $\$ 15,136$ por acre. Los costos más altos se incurrieron durante el primer año y se estima que fueron alrededor de $\$ 29,679$ por acre, basados en los costos de preparación, plantado de los catos, construcción de los enrejados, y prácticas culturales. Debido a que la fruta se puede cosechar a partir del segundo año, los costos acumulados declinaron de $\$ 29,679$ por acre en el primer año a $\$ 25,687$ por acre en el segundo, y a $\$ 15,136$ por acre en el tercer año. Los cactos requieren tres años para alcanzar madurez, así es que, los gastos acumulados de $\$ 15,136$ en el tercer año son amortizados sobre la vida del huerto (que se asumen son de 20 años) a una tasa de interés anual del cinco por ciento por un cargo anual fijo de alrededor de $\$ 1,215$, el cual se le carga a la empresa durante cada uno de los años de producción plena (del cuarto año en adelante). Por lo tanto, se estima que los costos de establecimiento de un huerto de cinco acres es de $\$ 75,680$ ( $\$ 15,136$ por 5). Esta cantidad no incluye los costos de capital de bienes comprados por el dueño.

Los costos e ingresos anuales del huerto maduro también están resumidos en el Cuadro 1 bajo el encabezamiento de "Años de Producción Plena." Basado en la asunción de que el huerto requiere tres años para alcanzar madurez, la producción plena ocurre del cuarto año en adelante. Se estima que los costos de mantenimiento en un año típico de un huerto en plena producción son de $\$ 10,127$ por acre. Hay un costo variable de pre-cosecha de $\$ 6,780$ (66.9\%) que cubre costos tales como de irrigación, aplicación de productos químicos, labor e intereses. Se estima que los costos de cosecha y mercadeo son de $\$ 950$, lo que representa el 9.4 por ciento del costo total de producción. Por lo tanto, los costos variables totales (costos variables de pre-cosecha más costos de cosecha y mercadeo) son de $\$ 7,730$, o 76.3 por ciento del costo total de producción. Los Costos Fijos, incluyen costos del dinero en efectivo, $\$ 555$ (5.5\%) y otros costos que no son de dinero en efectivo, $\$ 1,843$ (18.2\%), por un total que abarca el 23.7 por ciento del costo total. Asumiendo rendimientos de 19,000 libras por acre (90\% empacado) durante los años de producción plena implica un costo de producción de $\$ 0.53$ por libra. 
Basado en un rendimiento de 19,000 libras por acre y un precio promedio de venta de $\$ 1.35$ por libra, los ingresos totales serían de $\$ 25,650$ por acre. Esto resulta en ingresos netos para el productor de $\$ 15,523$ por acre $(\$ 25650$ menos $\$ 10,127)$. El punto de equilibrio entre ganancias y pérdidas es de $\$ 0.53$ por libra y 7,502 libras por acre respectivamente (Cuadro 2). Esto implica que, en base a las asunciones presentes, los precios o rendimientos tienen que declinar del promedio por más del 61 por ciento antes de que los ingresos sean negativos. Debido a las grandes fluctuaciones en rendimientos y precios, el Cuadro 3 muestra los ingresos netos por acre de varias combinaciones de precios y rendimientos. Es importante mencionar que aun cuando los precios bajan a $\$ 0.85$ por libra ( $37 \%$ debajo del promedio) y los rendimientos bajan a 17,000 libras por acre (11\% debajo del promedio), los productores todavía pueden obtener ingresos positivos.

El Cuadro 4 resume los costos de inversión (capital) y los cargos anuales de la empresa (recobro de capital) basados en los requerimientos para el establecimiento de un huerto de cinco acres de pitaya. Los costos totales de inversión se estiman en $\$ 109,830$. Esto representa los costos iniciales de operación de la empresa que es propiedad del productor $(\$ 34,150)$, tales como el costo de uso del vehículo, cortadoras, sistemas de irrigación, tubería principal y excavado del pozo, y costo de otras herramientas. Los costos de inversión también incluyen los costos de establecimiento del huerto, calculado anteriormente a ser de $\$ 15,136$ por acre, o \$75,680 por cinco acres. Los costos de inversión asumen que los bienes que se compran deben ser nuevos y deben ser usados exclusivamente en el huerto. La compra de equipos usados bajaría los costos de inversión pero los costos de reparación y mantenimiento se incrementarían. Se debe mencionar que el costo del terreno no está incluido en el costo de inversión porque se asume que la mayoría de los productores son dueños del terreno. Incluir el costo del terreno cusaría que el costo de inversión se incremente sustancialmente.

El costo anual de recobro de capital (costo de depreciación e interés) se estimó en $\$ 1,842$ por acre. Como se mencionó anteriormente, esto representa la cantidad anual que se carga a la empresa por la porción de activos fijos que se utilizan en la operación durante ese año.

\section{Conclusiones}

Los productores de frutas tropicales en el sur de Florida están en la busca de alternativas rentables para incrementar sus ganancias y para asegurarse que sus operaciones permanezcan rentables. Una fruta que es prometedora es la pitaya. Hay una gran demanda por este producto, especialmente entre los consumidores asiáticos, lo que es un buen augurio para los productores. Frutas vistas como "super frutas" tienen altos niveles de antioxidantes o nutrientes y se están volviendo muy populares entre los consumidores. Se espera que la demanda por pitaya (fruta dragón) se incremente porque se la ha calificado como "super fruta" debido a su alto contenido de antioxidantes, tal como el licopeno. Aún más, la fruta se ha convertido en la favorita de los chefs en los restaurants finos por su belleza y versatilidad.

Este estudio buscó proveer a los productores con información sobre costos de establecimiento y operación de un huerto de cinco acres con pitaya en el sur de Florida. Bajo las asunciones del análisis, el costo de establecimiento de tal huerto sería de $\$ 15,136$ por acre, o $\$ 75,680$ por cinco acres. Los costos totales de inversión (compra de todos los bienes de capital, los costos de establecimiento y mantenimiento por los tres primeros años de un huerto de cinco hectáreas) son estimados en $\$ 109,830$. Esta cantidad no incluye el costo/valor del terreno. Los costos totales de operación de un huerto de pitaya en plena producción (a partir del cuarto año) se estimaron en $\$ 10,127$ por acre. Con un precio promedio de $\$ 1.35$ por libra y un rendimiento vendible de 19,000 libras por acre, el ingreso promedio estimado fue de $\$ 25,650$ por acre, resultando en un ingreso neto (ganancia neta) de $\$ 15,523$ por acre. Esto representa un ingreso muy favorable, comparado con el de otras frutas tropicales, tal como el mango y los aguacates, que tienen un ingreso promedio de $\$ 1,500$ por acre.

A pesar del resultado favorable del análisis, se aconseja a los productores que actúen con cautela porque el mercado podría ser fácilmente sobreavastecido. Hay oportunidades para mejorar y hacer más eficiente el proceso de producción de pitaya usando tecnología avanzada (por ejemplo, la floración puede ser inducida usando luz artificial). Sin embargo, se debe conducir un análisis cuidadoso de costos e ingresos par ver si el incremento en la eficiencia de producción justifica los costos adicionales.

\section{Referencias}

Crane, J. 2009. Comunicación personal. Profesor Especialista en Frutas Tropicales, University of Florida, Tropical Research and Education Center, Homestead, FL.

Crane, J.H., and C.F. Balerdi. 2005. The Pitaya (Hylocereus undatus and other spp.) in Florida. http://miami-dade.ifas. ufl.edu/pdfs/tropical_fruit/THE\%20PITAYA $\% 20 \mathrm{in} \% 20$ Florida.pdf 
Crane, J.H., and C.F. Balerdi. 2009. Pitaya growing in the Florida home landscape. Electronic Data Information Source (EDIS) HS1068. UF/IFAS, Gainesville, FL. http:// edis.ifas.ufl.edu/HS1068

Gunasena, H.P.M., D.K.N.G. Pushpakumara, and M. Kariyawasam. 2006. Dragon fruit-Hylocereus undatus (Haw.) Britton and. Rose: Field manual for extension workers. Sri Lanka: Sri Lanka Council for Agricultural Policy. http:// www.worldagroforestry.org/downloads/publications/PDFs/ BC07324.PDF
Lobo, R., and G. Bender. 2008. Pitahaya field test yields preliminary results. In Small Farm News, Small Farm Program, Volume 2. Davis, CA: University of California at Davis.

Mizrahi, Y., A. Nerd, and P.S. Nobel. 1997. Cacti as crops. Horticultural Reviews 18: 321-346.

Steele, D., and J.H. Crane. 2006. The state of the Florida tropical fruit industry and the challenges growers face. Proceedings of the Florida State Horticultural Society 119: 7-8. 
Cuadro 1. Costos de establecimiento y producción de un huerto de cinco acres con pitaya en el sur de Florida

\begin{tabular}{|c|c|c|c|c|c|}
\hline & \multirow[b]{2}{*}{ Unidad } & \multicolumn{3}{|c|}{ Años de Establecimiento } & \multirow{2}{*}{$\begin{array}{c}\text { Años en } \\
\text { Producción Plena }\end{array}$} \\
\hline & & Año 1 & Año 2 & Año 3 & \\
\hline Rendimientos Estimados & lbs/acre & 0 & 10,000 & 15,000 & 19,000 \\
\hline Precios Estimados & $\$ / \mathrm{lb}$ & 0 & 1.35 & 1.35 & 1.35 \\
\hline Ingresos Totales & & 0 & 13,500 & 20,250 & 25,650 \\
\hline \multicolumn{6}{|l|}{ COSTOS } \\
\hline \multicolumn{6}{|l|}{ Costos de Preparación } \\
\hline Preparación & & 500 & & & \\
\hline Construcción y materiales para el enrejado & & 18,590 & & & \\
\hline Plantas y costos de plantación & & 3,360 & & & \\
\hline \multicolumn{6}{|l|}{ Costos Variables de pre Cosecha } \\
\hline Irrigación & & 50 & 50 & 50 & 50 \\
\hline Fertilización & & 1,410 & 1,560 & 1,560 & 1,705 \\
\hline Herbicidas & & 388 & 400 & 412 & 424 \\
\hline Insecticidas & & 415 & 427 & 440 & 453 \\
\hline Fungicidas & & 28 & 29 & 30 & 31 \\
\hline Cortar hierbas & & 120 & 124 & 127 & 131 \\
\hline Costs de Labor (podas, fertilización, etc.) & & 3,102 & 3,195 & 3,291 & 3,390 \\
\hline Reparaciones & & 250 & 258 & 265 & 273 \\
\hline Intereses sobre los costos de pre cosecha (5\%) & & 288 & 302 & 309 & 323 \\
\hline \multicolumn{6}{|l|}{ Costos de Cosecha y Transporte } \\
\hline Recolección, empaque y transporte & & 0 & 500 & 750 & 950 \\
\hline Total de Costos Variables & & 28,501 & 6,844 & 7,234 & 7,730 \\
\hline \multicolumn{6}{|l|}{ Costos Fijos Anuales de Dinero en Efectivo } \\
\hline Impuestos a la propiedad & & 50 & 52 & 53 & 55 \\
\hline Terreno ( precio de renta) & & 500 & 500 & 500 & 500 \\
\hline \multicolumn{6}{|l|}{ Otros Costos Fijos Anuales } \\
\hline Equipo de irrigación & & 203 & 203 & 203 & 203 \\
\hline Otras maquinarias y equipo & & 425 & 425 & 425 & 425 \\
\hline Pago de costos de establecimiento & & & & & 1,215 \\
\hline Total de Costos Fijos & & 1,178 & 1,180 & 1,181 & 2,397 \\
\hline Intereses Sobre Costos de Establecimiento & & 0 & 1,484 & 1,284 & 0 \\
\hline TOTAL DE COSTOS & & $-29,679$ & 9,508 & 9,699 & 10,127 \\
\hline RETORNOS NETOS & & 29,679 & 3,992 & 10,551 & 15,523 \\
\hline Costos Acumulados de Esablecimiento & & 29,679 & 25,687 & 15,136 & \\
\hline
\end{tabular}


Cuadro 2. Precio y rendimiento de equilibrio entre ganancia y pérdida

\begin{tabular}{|lcc|}
\hline & Unidades & Total \\
\hline Precio de equilibrio & dólar por libra & 0.53 \\
Rendimiento de equilibrio & libras & 7,502 \\
\hline
\end{tabular}

Cuadro 3. Ingresos netos estimados a diferentes niveles de precios y rendimientos

\begin{tabular}{|lccccc|}
\hline & \multicolumn{4}{c|}{ Rendimiento (lbs/acre) } \\
\cline { 2 - 6 } Precio $(\$ / \mathrm{lb})$ & 17,000 & 18,000 & 19,000 & 20,000 & 21,000 \\
\hline 0.85 & 4,491 & 5,291 & 6,091 & 6,891 & 7,691 \\
1.00 & 7,041 & 7,991 & 8,941 & 9,891 & 10,841 \\
1.35 & 12,991 & 14,291 & 15,523 & 16,891 & 18,191 \\
1.50 & 15,541 & 16,991 & 18,441 & 19,891 & 21,341 \\
2.00 & 24,041 & 25,991 & 27,941 & 29,891 & 31,841 \\
2.50 & 32,541 & 34,991 & 37,441 & 39,891 & 42,341 \\
\hline
\end{tabular}

Cuadro 4. Costos de inversión en un huerto de cinco acres y recobro del capital por año y por acre

\begin{tabular}{|lcccc|}
\hline Artículos de Inversión & Precio de Compra* & Vida Util & Tasa de Interés & Recobro de Capital \\
\cline { 2 - 5 } & $(\$)$ & (Años) & $(\%)$ & $(\$ /$ acre) \\
\hline Vehículo utilitario & 9,500 & 15 & 0.05 & 183 \\
Cortadora (50pulgadas) & 7,000 & 15 & 0.05 & 135 \\
Pulverizador de 200galones y accesorios & 3,500 & 15 & 0.05 & 67 \\
Sistema de irrigación, tuberia, bomba, pozo & 12,650 & 20 & 0.05 & 203 \\
Otros equipos y herramientas & 1,500 & 10 & 0.05 & 39 \\
Costos de establecimiento y mantenimiento del huerto & 74,870 & 20 & 0.05 & 1,215 \\
Inversión total en un huerto de cinco acres & 74,870 & & & 1.842 \\
\hline * Excluye el costo del terreno. & & & & \\
\hline
\end{tabular}

